\title{
Utilizing Pharmacogenetics in Psychiatry: the Time Has Come
}

\author{
David Durham
}

Published online: 23 January 2014

(c) Springer International Publishing Switzerland 2014

Psychiatry remains the most subjective field in medicine. This has probably been the greatest barrier to improving the accuracy of diagnosis, quantifiably reducing adverse drug events, and substantially improving treatment outcomes. The utilization of pharmacogenetic technology in psychiatry is the first clinical modality of its kind to increase both the accuracy and the precision of prescribing psychotropic medications while simultaneously reducing adverse drug events and deaths.

The Centers for Medicaid and Medicare Services CMS) estimate that adverse drug events (ADEs) in the USA cost about US\$289 billion [1], a figure that does not consider data from numerous private insurance companies [2]. Over 770,000 people are injured or die each year in hospitals from ADEs. Adverse drug reactions are estimated to be responsible for an estimated 100,000 deaths every year and range from the fourth to the sixth leading cause of death in the USA [2, 3]. Studies of geriatric outpatients have found that the percentage of potential clinically significant adverse drug reactions ranges from 6 to $25 \%$ [4]. An estimated $17 \%$ of the top 200 prescribed drugs in 2012 have a US Food and Drug Administration (FDA) pharmacogenetic warning in the package insert. These numbers grow larger each year. There is good information, dating back more than a decade, that psychiatric medications have, for some time, been among the most common medications found in deaths caused by the adverse effects of prescription drugs. A report [5] published in 2005 by Drs. Paulozzi and Annest at the Centers for Disease Control (CDC), titled 'Unintentional Poisoning Death: United

D. Durham $(\bowtie)$

The University of New Mexico, School of Medicine,

Albuquerque, NM, USA

e-mail: ddurham@sageclinic.org
States 1999-2004', found that opioids were, by far, the most common medication associated with death caused by prescribed drugs during this period-at $75.2 \%$. The authors found that the second most common prescribed medication was benzodiazepines - at $29.4 \%$ - and the third was antidepressants-at $17.6 \%$. The CDC's on-line 'Drug Overdose in the United States: Fact Sheet' reports that in 2010, of the 38,329 drug overdose deaths in the USA, 22,134 $(60 \%)$ were related to prescription drug overdoses, and 6,497 (30 \%) involved benzodiazepines. In the same CDC report, the authors note that in 2011, approximately 501,207 emergency department visits were related to anti-anxiety and insomnia medications [6, 7]. It is noteworthy to mention that all of the medications in the benzodiazepine family, and most of the medications in the sedative-hypnotic family, are principally metabolized by the cytochrome P450 (CYP) enzyme system.

In October 2013, an article published in JAMA Psychiatry addressed the mounting concern that the increased mortality risk among patients with psychiatric illness may be worsened by psychopharmacological agents. The authors analyzed patients participating in psychopharmacology clinical trials, using the US Food and Drug Administration (FDA) Summary Basis of Approval (SBA) reports of new drug applications and supplemental applications for 28 psychiatric medications approved between 1990 and 2011. Their analysis supported previous studies, which showed that having a psychiatric diagnosis itself increases the overall mortality risk. However, the authors found that the mortality risk was not increased when patients were assigned to psychotropic agents rather than placebo, with the exception of heterocyclic antidepressants [8].

Pharmacogenetics is rooted in the CYP system. CYPs are the metabolic factories in the liver and the mucosal 
surface of the intestinal tract. They can be likened to the body's waste management system for drugs, toxins, and cellular waste products. Scientists have also likened them to cellular highways for drugs. CYPs can be conceptualized as a combination of both.

There are four CYPs that metabolize almost all hepatic drugs. They are CYP2D6, CYP2C9, CYP2C19, and CYP3A4/5. Importantly for psychiatric pharmacogenetics, the first three of these-CYP2D6, CYP2C9, and CYP2C19-metabolize approximately $70 \%$ of all psychotropic medications. Genetic variations in CYPs impact more patients than common genetic disorders. These include trisomy 21 (also known as Down syndrome), which occurs in 1 out of about 700 US births; cystic fibrosis, which occurs in about 1 in 31 people or 1,000 babies born each year; and familial breast cancer (BRCA1 and BRCA2), which occurs in about 1 in 8 women. Data from the Mayo Clinic, the Genelex Corporation, and the National Institute of Health's Clinical Pharmacogenetics Implementation Consortium (CPIC) estimate that $70 \%$ of people have a variation in at least one of the principal CYP enzymes and do not metabolize medication normally $[9$, 10]. This individual variability is what contributes to adverse drug reactions.

In 2011, the Mayo Clinic completed a retrospective study of 60 outpatient adult psychiatric patients at the Hamm Clinic in St. Paul (MN, USA) [11]. The results showed a $31.2 \%$ reduction in depressive symptoms in those patients who received pharmacogenetic testing, compared with only a $7.2 \%$ reduction in depressive symptoms in those who were not tested $(p<0.02)$. A larger follow-up replication study of 200 outpatient psychiatric patients, by a Mayo Health System affiliate in La Cross (WI, USA), showed a $44.8 \%$ reduction in depressive symptoms with pharmacogenetic testing, compared with a $26.4 \%$ reduction without testing. This study yielded much greater power in its results, with $p<0.001[11,12]$. The vehicle of both studies depended on using pharmacogenetic testing at the subject's initial visit, for psychiatric medication selection and modification, and to help guide treatment.

A more recent study by a research group at the University of Illinois Department of Psychiatry (in Chicago, IL, USA), completed in 2012 and published in 2013, followed 96 patients over 1 year. The study was rather interesting in that it prospectively looked at the clinical impact of pharmacogenetic testing and then retrospectively looked at health utilization costs. The authors utilized proprietary software by AssureRx to identify those patients at increased risk of adverse drug reactions, and found that those who were slow metabolizers in either the 2D6 or 2C19 enzymatic pathways had $69 \%$ more total health care visits, $67 \%$ more general medical visits, greater than threefold more medical absence days, and greater than fourfold more disability claims [13].

One of the more elegant studies was by Chou et al. [14]. This study was published in 2000 — the metaphorical 'Dark Ages' of clinical pharmacogenetics-before FDA approval of the first clinical pharmacogenetic technology in 2002: the Roche Pharmaceuticals Amplichip. Chou's team studied 100 psychiatric inpatient adults at Eastern State Hospital in Lexington (KY, USA). All patients were genotyped for CYP2D6 and individually tracked for adverse drug events, hospital stays, and total costs over a 1-year period. The research team observed three dramatic trends. The first was a trend toward greater numbers of ADEs from psychotropic medications as one moved from patients with ultrarapid CYP2D6 activity (UMs) to patients with absent CYP2D6 activity, i.e. poor metabolizers (PMs). The second trend was the average cost of treating patients with extremes in CYP2D6 activity (UMs and PMs), which was US $\$ 4,000$ to US $\$ 6,000$ per year greater than the cost of treating normal and intermediate metabolizers (IMs). The third trend was that CYP2D6 PMs had longer hospital stays. This study demonstrated that patients who are CYP2D6 poor metabolizers and CYP2D6 ultrarapid metabolizers have more adverse drug events and longer hospital stays, and are simply more expensive to treat. It also demonstrated that proper application of pharmacogenetic technology is both a simple and a precise answer to help reduce ADEs, reduce costs, and better manage hospital stays.

These studies have shown improved clinical outcomes attributable to pharmacogenetic testing in clinical practice. Some have demonstrated reduced cost with the technology's appropriate utilization. Like all studies, these studies have had limitations. Their sample sizes were all small. Study subjects likely received more attention and subsequently closer follow-up, which may have contributed to some small improvement in depressive symptoms. Thus, there remains a clear need for a large randomized clinical trial to address both the clinical efficacy and the economic impact of pharmacogenetic testing in clinical practice.

In October 2013, CMS approved the first multicenter observational cohort study of 250,000 patients, called the Diagnosing Adverse Drug Reactions Registry (DART). This registry is sponsored by Renaissance $\mathrm{Rx}$ and is not limited to psychiatry but spans all medical specialties. Its purpose is to assess whether use of pharmacogenetic data results in a meaningful change in a subject's drug or dose. Importantly, it also seeks to evaluate the relationship between adverse drug reactions (ADRs) and genotype, and to assess if there is a relationship between healthcare utilization-specifically, emergency department visits and hospitalizations-and ADRs [15]. This is CMS's entry into the pharmacogenetic realm and the 'buccinator novo 
temporis ${ }^{1}$ for more robust pharmacogenetic studies. Large randomized clinical trials are, undoubtedly, around the corner.

Psychotropic medications are now the most prescribed drugs in medicine, despite a repository of data clearly revealing that they are far from benign and are likely overprescribed. Just as compelling are the data showing that nearly half of the people who are prescribed these medications do not respond well to treatment. Many of these non-responding patients often require complex psychotropic cocktails, which dramatically compound the potential for adverse drug events. Coupled with skyrocketing healthcare utilization costs, it seems the perfect opportunity to introduce psychiatric pharmacogenetic technology, not only to the broader psychiatric community but also to the broader medical community. The time is right for a large randomized clinical trial, as the time for practicing psychiatrists to improve our methods and safety of prescribing-using an available technology to give the right medicine at the right dose at the right time-is long overdue.

Acknowledgements and Disclosures The author has no conflicts of interest that are directly relevant to the content of this article.

\section{References}

1. Classen DC, Pestotnik SL, Evans RS, et al. Adverse drug events in hospitalized patients. JAMA. 1997;277(4):3016.

2. Cullen DJ, Bates DW, Small SD, et al. The incident reporting system does not detect adverse drug events: a problem for quality improvement. J Qual Improv. 1995;21(10):5418.

3. Lazarou J, Pomeranz BH, Corey PN. Incidence of adverse drug reactions in hospitalized patients: a metaanalysis of prospective studies. JAMA. 1998;279(15):1200-5.
4. Tulner LR, Frankfort SV, et al. Drug-drug interactions in a geriatric outpatient cohort: prevalence and relevance. Drugs Aging. 2008;25(4):343-55.

5. Centers for Disease Control and Prevention. Unintentional poisoning deaths-United States, 1999-2004. MMWR Morb Mortal Wkly Rep. 2007;56(5):93-6.

6. Centers for Disease Control and Prevention. Drug overdose in the United States: fact sheet. http://www.cdc.gov/homeandrecreational safety/overdose/facts.html.

7. Substance Abuse and Mental Health Services Administration. Highlights of the 2011 Drug Abuse Warning Network (DAWN) findings on drug-related emergency department visits. The DAWN Report. Rockville: US Department of Health and Human Services, Substance Abuse and Mental Health Services Administration; 2013. http://www.samhsa.gov/data/2k13/DAWN127/ sr127-DAWN-highlights.htm.

8. Khan A, Faucett J, Morrison S, Brown WA. Comparative mortality risk in adult patients with schizophrenia, depression, bipolar disorder, anxiety disorders, and attention-deficit/hyperactivity disorder participating in psychopharmacology clinical trials. JAMA Psychiatry. 2013;70(10):1091-9.

9. Relling MV, Klein TE. CPIC: Clinical Pharmacogenetics Implementation Consortium of the Pharmacogenomics Research Network. Clin Pharmacol Ther. 2011;89(3):464-7.

10. Mrazek D. Psychiatric pharmacogenomics. New York: Oxford University Press; 2011.

11. Mrazek D. Mayo Clinic Research Symposium-clinical implementation of psychiatric pharmacogenomic testing: characterizing and displaying genetic variants for clinical action; 2011.

12. Hall-Flavin DK, Winner JG, Allen JD, Jordan JJ, Nesheim RS, Snyder KA, et al. Using a pharmacogenomic algorithm to guide the treatment of depression. Transl Psychiatry. 2012;2:e172.

13. Winner J, Allen JD, et al. Psychiatric pharmacogenomics predicts health resource utilization of outpatients with anxiety and depression. Transl Psychiatry. 2013;3(3):e242.

14. Chou WH, Yan FX, et al. Extension of a pilot study: impact from the cytochrome P450 2D6 polymorphism on outcome and costs associated with severe mental illness. J Clin Psychopharmacol. 2000;20(2):246-51.

15. Diagnosing Adverse Drug Reactions Registry (DART). ClinicalTrials.gov Identifier: NCT01970709. http://www.clinicaltrials. gov/ct2/show/NCT01970709?term=Renaissance.

${ }^{1}$ Latin for 'trumpeter of new times'. 